Publié dans la revue Éducation et société, 2008, n² 22, pp. 29-42.

\title{
De l'usage des technologies de l'information et de la communication comme apprentissage créatif
}

\author{
Francis Jauréguiberry
}

«La science découvre, l'industrie applique et l'homme suit ». Cette affirmation, à la gloire de la science et de la technologie, qui figurait en exergue de la plaquette présentant l'exposition universelle de Chicago de 1933, pourrait résumer le type d'approche qui, à la fin de la décennie 1970 et durant toute celle des années 1980, a dominé les discours et les études sur ce que l'on appelait alors les nouvelles technologies de l'information et de la communication (NTIC). Comme l'industrie l'avait été en son temps, les NTIC furent investies d'une capacité à définir un nouveau monde. Leur caractère novateur et presque magique (car permettant la réalisation partielle d'un attribut de Dieu, l'ubiquité) ne fit que renforcer cette vision des choses. Cette approche, qui pourrait être qualifiée de déterminisme technique ou de technicisme, se situe toujours en aval de la conception de la technologie. Elle consiste, par l'observation de la mise en place des technologies, à évaluer leurs effets sur les comportements individuels et collectifs et, partant, sur l'organisation et le changement social. La question posée est en définitive de savoir comment la technique influe sur le socioculturel, l'économique et le politique.

Une dimension éducative implicite accompagne cette conception des choses : pour aller dans le sens du «progrès » et du « développement», les NTIC doivent être «bien utilisées ». Une «bonne utilisation » revient à suivre les prescriptions des concepteurs et à respecter les modalités de fonctionnement des objets. Celles-ci ne sont pas le fruit du hasard, mais de milliers d'heures de travail et de sommes d'ingéniosité. L'usager ne doit pas rompre avec cette chaîne vertueuse du progrès mais doit, lui aussi, y participer. Un bon utilisateur est donc un usager qui fait un effort d'apprentissage technique. Dans cette perspective, le mode d'emploi est pensé comme outil pédagogique et comme lien entre l'ingénieur savant et l'usager profane. L'usager ne sait pas : il doit apprendre. Le savoir descend, du scientifique à l'ingénieur et de l'ingénieur à l'usager. L'objet technique est une invite à la culture technique.

Les cahiers des charges des premières études d'usage des NTIC, commanditées au début des années 1980, allaient tous dans ce sens. Il était implicitement demandé aux sociologues de mesurer les écarts entre les utilisations attendues (les «bons usages ») et les utilisations observées. L'idée était de repérer les «zones de résistance » ou de «mauvaises utilisations" afin d'améliorer les modes d'emploi ou d'organiser la formation des usagers. Mais, quelques mois seulement après le lancement de ces études, la surprise des commanditaires fut de taille. Presque toutes les observations mettaient en effet l'accent sur un phénomène négligé à la fois par les ingénieurs (centrés sur le fonctionnement de l'appareil) et par les économistes (pour lesquels le modèle diffusionniste de Rogers restait le plus adéquat): les apprentissages des nouvelles machines à communiquer ne sont ni disciplinés ni passifs mais désordonnés et actifs. 
Les modes d'emploi sont certes lus, mais les usages débordent les prescriptions des concepteurs. Les utilisateurs bricolent, bidouillent, s'approprient l'appareil technique en fonction de leur culture, de leurs savoirs, de leurs besoins et, ce faisant, en viennent à inventer de nouveaux usages non prévus par les concepteurs.

De façon inaugurale, les nombreuses études menées dès le début des années 1980 sur le vidéotex (Minitel) montrent de façon exemplaire comment un terminal de communication, primitivement conçu pour uniquement consulter des banques de données, a été concrètement aussi utilisé à des fins de divertissement et de rencontres virtuelles (messageries). Il est à noter la dimension fortement interactive et collaborative de ces détournements. Ainsi la messagerie, en particulier «rose», résulte-t-elle du détournement d'une possibilité de jouer aux échecs en ligne avec des inconnus. Sur l'écran figurait l'échiquier, avec cases et pièces, et une ligne où chacun pouvait écrire jusqu'à une dizaine de mots afin de commenter la partie. Quelques semaines seulement après la mise en ligne, on s'est aperçu que les pièces restaient étrangement fixes mais que, par contre, les mots ne cessaient de défiler sur la ligne de communication ouverte... Pour la première fois, des inconnus pouvaient s'écrire sous forme anonyme ce que bon leur semblait, sans autre restriction que leurs attentes, désirs ou phantasmes. L'échiquier disparut de l'écran et le dialogue en temps réel entre inconnus resta. Une partie de ce qui allait faire le succès du Minitel est le pur produit d'un usage inventif des utilisateurs, au-delà de tout ce qui avait primitivement été prévu. C'est dans leur collaboration créative que les utilisateurs avaient appris quelque chose qui échappait de loin à ce qui était préalablement donné à savoir.

\section{L'écart entre les usages prescrits et les usages effectifs}

Les études de Baltz, Boullier, Charon, Cherki, Jouët, Mallein, Marchand, de Gournay, Mercier, Scardigli et Toussaint semblent s'accorder pour dégager la notion d'usage dans sa capacité à signifier l'autonomie des pratiques. La grande liberté de ces premiers chercheurs envers les commanditaires de leurs études mais aussi envers le structuralisme encore dominant à l'époque en France, leur sensibilité à la montée de l'individualisme et à la contestation des cadres traditionnels de l'action, ne sont sans doute pas étrangères à ce paradigme de l'autonomie. Au demeurant, celui-ci est également observable dans d'autres secteurs de la sociologie à un moment où la discipline hésite face à la crise de l'idée même de société. La remise en cause de l'évolutionnisme, la critique de l'idéologie du progrès, la valorisation de la réalisation de soi et déjà l'idéologie de la communication invitent par ailleurs à être particulièrement réceptif aux résistances à l'ordre technologique. Ce n'est toutefois pas la technologie en soi qui est dénoncée (bien que certaines technologies le soient effectivement par ailleurs : voir le débat sur le nucléaire), mais ses usages prescrits ou, si l'on préfère, l'image déterministe qu'ingénieurs et planificateurs leur font revêtir. Les premières expérimentations des NTIC font apparaître qu'il n'y a, de la part de leurs promoteurs institutionnels, «aucune considération de besoin, mais des stratégies politiques et économiques dans lesquelles les acteurs n'ont aucun droit de regard et encore moins de parole » (Laulan 1985, 80).

On voit comment la critique du déterminisme technique est ici doublée de celle du pouvoir économique ou, selon les auteurs, du pouvoir technocratique. Face aux «impératifs de rentabilité des industriels et des décideurs engendrant une sorte de violence (viol) des utilisateurs », ceux-ci «résistent» (Laulan 1985, 30). Par 
« résistance », Laulan entend « la réaction multiple, diverse, créatrice et toujours active que les citoyens, les utilisateurs, les publics apportent aux offres technologiques qui leur sont faites » (Laulan 1985, 9). «Corrélative de l'impérialisme du progrès et du rendement, la résistance renvoie à un autre type de consensus social, où le bonheur n'est pas imposé ni décidé, où l'avenir n'est pas domestiqué, où l'existence sociale n'est pas planifiée » (Laulan 1985, 11). C'est sur cette image de l'utilisateur résistant, actif et inventif que va se développa la sociologie des usages de ce qui fut un temps appelée «l'école française des usages ».

\section{Du bricolage au détournement}

Tous les chercheurs de cette « école » firent, à un moment ou à un autre, référence à Michel de Certeau. Le vocabulaire qu'ils emploient y renvoie implicitement lorsqu'il n'est pas cité. Que ce soit Jouët, Baboulin et Gaudin, Toussaint et Mallein, de Gournay ou Charon et Cherki lorsqu'ils décrivent « les manières de faire » avec le magnétoscope, le «bricolage » de la micro-informatique ou «l'appropriation active » du Minitel. Que ce soit Boullier (1984) ou Mercier (1984) lorsqu'ils parlent au même moment de « détournements de l'usage normalisé ». Que ce soit Perriault $(1989,14)$ lorsque, contre les technologues «qui ne voient que deux sortes de gens : ceux que se servent bien des appareils et ceux qui s'en servent mal», il pose que les usagers développent toujours « des déviances, des variantes, des détournements et des arpèges ». Que ce soit Laulan (1985, 83-84) lorsqu'elle décline la «résistance aux systèmes d'information » en « inventions de procédures » et en « détournements » ou encore Jauréguiberry $(1989,6)$ lorsqu'il décrit les «squats du visiophone réalisés au moyen d'explorations, art de faire et procédures inédites », la référence à de Certeau est omniprésente. Et ce n'est sans doute pas un hasard : tous ces chercheurs procèdent à la fois d'une tradition critique du pouvoir et d'une volonté de penser l'acteur social dans son autonomie. Pour eux, l'approche que fait de Certeau des pratiques quotidiennes est doublement séduisante. Elle permet à la fois d'aborder les usages des NTIC avec une sociologie attentive aux usagers pensés non plus seulement comme de simples consommateurs passifs mais aussi comme des acteurs et d'échapper à la vision totalisante et bien pessimiste « d'enfermement des pratiques par le pouvoir » à laquelle conduisent à la même époque les travaux dominants de Bourdieu et de Foucault. De Certeau se préoccupe en effet moins de savoir comment le pouvoir joue avec les faibles que de décrire comment ceuxci se jouent de la discipline (étudiée par Foucault) et de la domination (étudiée par Bourdieu). Ce que relèvent ces chercheurs au moment de définir leur objet — et non plus terrain - d'étude, c'est la façon dont de Certeau pose le fait que «les usagers bricolent avec et dans l'économie culturelle dominante les innombrables et infinitésimales métamorphoses de sa loi en celle de leurs intérêts et de leurs règles propres » (de Certeau 1980, 13).

« À une production rationalisée, expansionniste autant que centralisée, bruyante et spectaculaire, correspond une autre production, qualifiée de "consommation" : celle-ci est rusée, elle est dispersée, mais elle s'insinue partout, silencieuse et quasi invisible, puisqu'elle ne se signale pas avec des produits propres mais en manières d'employer les produits imposés par un ordre économique dominant » (de Certeau 1980, 11, souligné par l'auteur). Avec lui, apparaît donc la possibilité de penser l'acteur social dans son autonomie autrement que sous la forme d'un militant mu par une idéologie et s'inscrivant dans une stratégie. Entre le militant et l'aliénation, l'usager est replacé dans 
sa capacité d'acteur. L'invention du quotidien ne se fait pas avec de grandes mobilisations, qui ne font que reproduire le pouvoir sous d'autres formes, mais par le biais de micro-résistances visant au contraire à lui échapper : "Le quotidien s'invente avec mille manières de braconner » (de Certeau 1980, 10). "L'école française des usages » s'empara de cette image des usagers décrits comme des «producteurs méconnus, poètes de leurs affaires, inventeurs silencieux de sentiers propres dans les jungles de la rationalité fonctionnaliste » (de Certeau 1980, 19) pour étudier comment «les procédures populaires jouent avec les mécanismes de la discipline et ne s'y conforment que pour les tourner» et pour montrer comment «ces manières de faire constituent les mille pratiques par lesquelles des utilisateurs se réapproprient l'espace organisé par les techniques de la production socioculturelle » (de Certeau 1980, 14).

Pendant quelques années, la quasi-totalité des sociologues des usages des NTIC vont se reconnurent dans ce type d'approche. Délaissant les approches quantitatives, ils s'appliquèrent à repérer les manières de faire, prendre le temps d'observer, d'écouter et de revenir sans cesse sur les formes d'usage afin de les modéliser. Leur sociologie est donc qualitative et nécessite dans tous les cas une forte implication des chercheurs. Il leur faut en effet intervenir pour constater, intervenir pour vérifier, intervenir pour comprendre. Car à partir du moment où l'on parle de «braconnages », de « détours » et de « pratiques de résistance », il faut s'attendre à ne pas pouvoir les lister d'avance. Et si ces usages se rapportent de surcroît à des techniques nouvelles, il faudra par définition s'immiscer pour observer. C'est pour cette raison que les méthodes employées par les sociologues des usages sont toutes intervenantes : il s'agit d'observer en situation, d'écouter attentivement, d'interviewer longuement.

L'approfondissement des problématiques va confronta toutefois progressivement l'ensemble de ces chercheurs à une même question : est-ce que l'art de faire est une façon de faire avec en s'accommodant, à l'image du bricoleur qui adapte comme il peut ce dont il dispose parce qu'il n'a pas le choix, ou s'agit-il de résister en détournant, à la manière du judoka qui, se sachant plus faible que lui, se sert de la force de son adversaire pour lui faire perdre pied ?

Dans le premier cas, nous ne sommes jamais que dans une version francisée des études américaines en uses and gratifications. Dans le second, la résistance des usagers conduit plutôt vers une problématique d'un acteur social plus critique. Les réponses à cette question firent éclater «l'école française des usages » qui, pendant quelques années, avait donné une image suffisamment unie pour qu'elle puisse être ainsi nommée à l'étranger. Certains, devant la raréfaction des expérimentations de France Télécom et la forte demande en aide au développement de structures privées se recyclèrent sans difficulté dans le conseil. Quelques-uns se focalisèrent sur un travail de description chaque fois plus minutieux des procédures d'usage au point qu'on peut raisonnablement douter qu'il s'agisse encore de sociologie (les ethnométhodologues ne réclament au demeurant pas ce label). D'autres enfin optèrent pour approfondir l'idée d'une dimension critique des usages en posant que sa dimension non stratégique n'est pas pour cela synonyme de passivité et d'inefficacité en termes de changement social.

\section{Représentations d'usage et significations pour les usagers}

Un produit peut à la fois très bien marcher techniquement et tomber en panne économiquement. Pour peu qu'une publicité désastreuse n'ait pas accompagné son lancement, deux raisons peuvent alors expliquer sa faillite commerciale. Soit son prix 
de vente semble trop élevé au consommateur pour les satisfactions qu'il compte en tirer, soit ce produit, au prix raisonnable, se heurte à un problème d'acceptation : l'usager, oublié dans sa spécificité lors de la conception du produit, se venge. La science, la technologie et l'économie étaient au rendez-vous, le social et ses acteurs pas. Certes, l'essentiel du rôle des ingénieurs-concepteurs est de résoudre des problèmes d'ordre technique. Intermédiaires entre les découvertes scientifiques et les systèmes techniques, ils se doivent de traduire le théoriquement possible en pratiquement faisable. Mais leur horizon d'interrogation sur les finalités de leur produit s'en trouve le plus souvent réduit. Leur logique de concepteurs suit en effet le droit fil de la résolution de problèmes techniques précis dans un environnement donné de contraintes physiques. Le social n'est entrevu que comme un récepteur muet et supposé avide de nouveautés. La seule dimension hétérogène à la technique qui intervient (souvent tardivement et presque toujours sous forme d'un rappel à l'ordre) est d'ordre économique : le scientifiquement possible doit devenir non seulement techniquement mais aussi économiquement faisable.

Le danger d'une telle logique de conception fermée semble évident : emportés par l'effort et souvent la passion de résolution de problèmes purement techniques, les concepteurs produisent de réels bijoux technologiques malheureusement inadaptés à la culture de ceux qui auront à s'en servir. Alain Gras, Caroline Morico, Sophie PoirotDelpech \& Victor Scardigli (1994) ont ainsi montré comment l'informatisation dans le monde aéronautique avait été différemment vécue selon les catégories de travailleurs qu'elle concernait et au premier chef les pilotes de ligne et les contrôleurs aériens. Pour les contrôleurs aériens, l'innovation est assimilée à un processus coopératif. L'innovation se fait avec eux : ils participent, en les testant, à la mise au point des nouveaux dispositifs d'aide au contrôle. Les contacts fréquents qu'ils entretiennent avec les concepteurs de systèmes les conduisent à intégrer en partie les représentations des concepteurs et ceux-ci s'imprègnent en retour de l'environnement humain des contrôleurs. Les négociations dont parle l'école de la traduction intègrent donc ici l'utilisateur. L'innovation est finalement le résultat d'une co-gestion de la recherche et se soldexa au final par une, son adoption. C'est presque l'opposé qui se passe avec les pilotes de ligne. L'informatisation des cockpits (celui du A 320 fait en la matière figure de pionnier) est portée par des ingénieurs formés à la seule rationalité technicoopérationnelle et se heurte d'entrée à la vision humaine (si ce n'est potentiellement héroïque) des pilotes. "Les ingénieurs opèrent un clivage de l'univers entre les créateurs et les autres hommes, pilotes aussi bien que passagers » (Gras \& al. 1994, 229). Alors que les pilotes comptent sur leur savoir-faire, leur expérience et leur intuition pour faire face aux aléas du pilotage et en particulier aux situations de danger, les ingénieurs veulent au contraire informatiquement tout prévoir afin de ne laisser aucune chance à l'erreur. Erreur que les pilotes entendent immédiatement comme humaine... L'antagonisme de ces deux cultures se solda par une forte résistance et une mobilisation de la profession contre l'innovation. Au total, on voit comment la connaissance de la culture des groupes d'appartenance est importante au moment de comprendre les types d'usages d'une innovation, la résistance étant l'un d'entre eux.

Plaidant pour une sociologie de l'appropriation, Alain Gras \& al (1994, 261-262) signalent que celle-ci se décline à trois niveaux. D'abord, au niveau individuel, «l'utilisateur agit de façon à ce que l'innovation convienne à sa personnalité : il l'intègre dans ses schèmes perceptivo-moteurs familiers, ses habitudes de travail et son 
expérience antérieure (...). Plus globalement, il organise l'ensemble de ses objets techniques quotidiens pour leur donner un sens personnel, lié à la trame de sa propre vie : comme s'il réalisait une "mise en intrigue" de l'innovation, pour parler comme Paul Ricœur ». Ensuite au niveau du groupe d'appartenance, où les cultures de métier, des classes d'âge et de milieux jouent un rôle fondamental. Et enfin au niveau de la culture, « au sens géographique de culture régionale ou nationale ».

Les deux premiers niveaux se retrouvent souvent dans les recherches de «l'école française des usages » des années 1980. Ainsi, Mallein et Toussaint $(1994,318)$ notentils que «nos [10 ans de recherche] ont montré que l'insertion sociale d'une NTIC, son intégration à la quotidienneté des usagers, dépendaient moins de ses qualités techniques "intrinsèques", de ses performances et de sa sophistication que des significations d'usage projetées et construites par les usagers sur le dispositif technique qui leur était proposé. Face à un nouveau dispositif technologique, d'une manière plus ou moins explicite et consciente, les usagers effectuent une sorte de procès en légitimité de son usage. De l'issue de ce procès dépendront les conditions de l'intégration sociale de la nouvelle technologie de l'information et de la communication ». Jouët (2000), Chambat (1994) ou Proulx (2001) font exactement le même constat.

Le troisième niveau est par contre moins développé et pourrait être l'objet de futures études, par exemple européennes. Victor Scardigli (1993) a toutefois déjà bien montré comment une même innovation technique est différemment adoptée selon les données spécifiques, en particulier culturelles, du lieu. Ce qui donne par exemple « des visages fort différents à Berlin ou Paris, métropoles pourtant également remarquées pour leur ouverture à l'innovation. Et un contraste aussi fort oppose l'Émilie-Romagne et la Catalogne, alors même que ces régions constituent, pour leur propre pays, le modèle du développement économique ou d'exercice de la citoyenneté » $(1994,306)$. Ling, Julsrud \& Krogh (1997) décrivent de leur côté combien le vécu particulier qu'entretiennent les Norvégiens avec la nature, en particulier avec l'étendue, le silence et la forêt en se ressourçant au cours de longs week-ends, dans leurs hytte (cabane, chalet) les conduit à délaisser régulièrement leur téléphone mobile alors que, le reste du temps, ils en font un usage immodéré. À un tout autre niveau, celui des médias, les travaux comparatifs de Liebes \& Katz (1990), sur le décodage différencié de la série Dallas par les téléspectateurs de différents pays, relève de cette démarche. De façon plus générale, il n'y a aucune raison de penser que les désirs d'immédiat et de transparence communicationnelle soient universels.

\section{Les usages dans la dynamique de la vie quotidienne}

La nécessité de prendre en compte l'environnement quotidien des usagers pour comprendre leurs modes d'appropriation a donné lieu à une série de recherches ou plutôt de parties de recherches. Peu en effet se focalisèrent sur les modes de vie, les micro-cultures, les styles et modes de distinction, les genres liés au sexe ou à l'appartenance ethnique. Par contre, plusieurs cherchèrent à corréler types d'usage et appartenances. Ainsi, Chambat $(1994,259)$ peut-il noter que «les différences de taux d'équipements ou de fréquences d'usages ne sont que le révélateur des disparités de significations que revêtent les pratiques concernées pour les différents groupes sociaux. Mettre à jour ces significations nécessite le recours à des investigations plus qualitatives s'appuyant sur les tendances lourdes qui affectent l'évolution des modes de vie ». C'est ainsi que Gérard Claisse \& Frantz Rowe (1993) en France, comme John Dimmick, Scott 
Paterson \& Jaspreet Sikand (1996) aux États-Unis emploient une méthode originale : celle du carnet de bord où est notée chacune des communications émises ou reçues pendant une ou deux semaines. Leurs travaux mettent en relief de fortes discriminations sociales (par exemple, le nombre de messages par personne est deux fois plus élevé chez les cadres supérieurs que chez les ouvriers). De même, les célibataires utilisent deux fois plus le téléphone que les personnes vivant en famille. Hormis pour les célibataires, chez qui l'on n'observe pas de différences notables,=le sexe apparaît comme une variable très discriminante de l'usage du téléphone : les femmes téléphonent en moyenne deux fois plus que les hommes. Mallein \& Toussaint $(1994,317)$ rappellent que «le développement de nouveaux usages n'émerge pas ex nihilo (...). L'apparition de nouvelles pratiques se greffe sur le passé, sur des routines, sur des survivances culturelles qui perdurent et continuent à se transmettre bien au-delà de leur apparition ». De son côté, Gilles Pronovost insiste sur la nécessité de «situer les usages dans le contexte de l'ensemble de leurs pratiques culturelles ». Toute la difficulté réside dans l'opération d'évaluation de l'autonomie culturelle ou, plus simplement, de la mise en forme de l'originalité des modes de vie. À moins de décalquer les catégories classiques de la sociologie quantitative pour les recomposer (Juan 1995), il ne reste au sociologue qu'à se transformer en ethnologue s'il veut vraiment évaluer la prégnance culturelle dans les modes d'appropriation des NTIC.

C'est ce que certains firent, comme Alain Tarrius (1994) qui n'hésita pas à suivre pas à pas, durant plusieurs jours d'affilée, des usagers du téléphone mobile ou Anne Monjaret (1996) qui partagea des bureaux d'employés afin d'étudier les différences sexuées dans le traitement de la gestion des relations familiales par téléphone sur les lieux de travail. Si ce type d'étude demeure assez rare, il est par contre impossible de ne pas faire référence aux cultural studies britanniques, même si cette tradition de recherche n'a eu que peu d'écho en France. Au départ, on ne peut pas dire que les fondateurs se soient intéressés aux technologies de communication. L'exploration des réseaux de sociabilité et des modes de socialisation des classes populaires par Richard Hoggart, Stuart Hall, Raymond Williams et Edward \& Thompson était clairement associée à une problématique du pouvoir culturel et cherchait plutôt à dégager les mécanismes de résistance à la domination culturelle et à évaluer la capacité à établir des représentations alternatives à l'ordre imposé. La démarche était donc orientée vers une dénonciation de la domination... Ce n'est que dans les années 1980 que les cultural studies s'intéressèrent de près aux médias et, plus particulièrement, aux conditions de réception de la télévision. La méthodologie, de plus en plus fine, déployée pour l'observation du schéma de réception (encoding-decoding) conduisit à mettre l'accent sur l'importance du cadre domestique de réception, en particulier familial et amical. C'est dans la radicalisation de cette pratique méthodologique qu'Armande Mattelard \& Erik Neveu $(1996,39)$ situent le «tournant ethnographique » des cultural studies et l'incorporation, « de façon exponentielle, de chercheurs qui œuvrent sur les médias, les "gender studies", la géographie humaine, l'ethnicité, les loisirs et la consommation. » Le projet The household uses of information and communication technologies, mené par Silverstone, Morley, Dalhberg \& Livingstone (1989) s'inscrit parfaitement dans cet effort de recontextualisation des usages dans un but de compréhension de la complexité du phénomène de la réception. Les études de Haddon (1997), Ling, Julsrud et Krogh (1997) sur l'usage du téléphone portable adoptent le même type d'approche. 
Dans le même ordre d'idée, il faut signaler les approches en termes de filière d'emploi entre anciens et nouveaux outils de communication ou encore de déclinaison d'usages. Il s'agit d'évaluer en quoi et comment l'activité de communication réalisée à partir ou avec d'anciennes technologies conditionne l'emploi des nouvelles. Boullier (1992) montre par exemple comment le magnétoscope a été associé à la télévision (alors qu'au départ il avait été conçu pour effectuer des montages vidéo) ou le fax au téléphone. De son côté, Chambat $(1994,259)$, qui parle de «filières d'objets » et de «filières d'usages », rappelle comment l'adoption du micro-ordinateur s'est d'abord faite en référence à la machine à écrire et comment les premières utilisations du téléphone mobile se calquaient sur les pratiques connues du téléphone fixe. Promovost $(1994,382)$ préfère quant à lui parler de «continuum empirique d'usages », tandis que Mallein \& Toussaint $(1994,321)$ décrivent «l'accrochage du nouveau système technique à un système déjà implanté » ou de «fonctionnalité seconde à partir des usages préexistants ».

\section{Usage de la notion d'usage des NTIC}

Il fallait s'attendre à ce qu'une attention aussi poussée sur les formes d'usage soit un jour commercialement récupérée. La libéralisation du secteur des télécommunications et la concurrence entre opérateurs et entre fabricants de terminaux ont en effet, en quelques années seulement, transformé l'image des usagers des NTIC. Considéré comme « un citoyen plus ou moins prioritaire pour obtenir une connexion » jusqu'au début des années 1970, puis comme «clients » dans les années 1980, l'usager devient dans les années 1990 «un consommateur exigeant dont les besoins et les attentes font l'objet d'une attention particulière »(Carré \& Panico 1997). Depuis quelques années, les investissements dans les réseaux sont tels qu'il est devenu impensable que la demande en télécommunications arrête sa progression. Les scénarios de développement se fondent tous sur des prévisions à la hausse : celle-ci est en effet devenue économiquement indispensable.

La prise en compte des formes usages par les opérateurs se fait de deux façons. La première relève d'une démarche étroitement marketing de marché et au fil de l'eau. Il s'agit de repérer, parmi les usages, ceux qui ont le plus de succès (vision quantitative donc) pour calquer dessus des messages publicitaires. Rien n'empêche ensuite de procéder à des études plus poussées et de cerner des types d'usages plus restreints afin d'y associer des niches commerciales plus ciblées. La seconde façon est plus proche de la notion d'usage telle qu'elle a été définie plus haut, c'est-à-dire dans sa capacité à braconner, à détourner ou à imaginer en acte. L'expérience du Minitel a montré l'intérêt qu'il y eût à savoir observer l'usager dans son appropriation de l'objet ou du service. Car ce sont finalement les «braconniers » de Grétel qui ont imaginé le Minitel dans ce qui fit son succès. Si l'on en était resté aux premiers usages imaginés par ses ingénieurs concepteurs, le Minitel aurait sans doute connu le même désastre que, par exemple, en Allemagne. L'idée est donc de pouvoir intégrer des pratiques «d'usages porteurs » dans la «conception permanente » des objets techniques. Il faut pour cela faire appel à des sociologues menant des études qualitatives. Se pose alors la question de l'utilisation marchande, mais aussi, in fine, technologiste des études d'usage. C'est en effet en se basant sur ces études que les opérateurs proposent de nouveaux services afin de rentabiliser leurs paris technologiques et financiers. 
Et c'est exactement cette « récupération » que théorise puis propose sous forme de service, Philippe Mallein avec sa «Conception Assistée par l'Usage pour les Technologies d'Information et de Communication »(CAUTIC). Après avoir montré l'importance des habitudes et cultures des usagers dans la "mise en procès" qu'ils font de la nouvelle technique, Mallein en déduisit dès 1994 «qu'une telle façon de poser le problème de l'usage exprime une nouvelle forme d'interaction offre-demande relative aux NTIC » (Mallein \& Toussaint 1994, 318). Ce fut le début d'un cheminement qui conduisit ce chercheur, tout à fait représentatif de l'école française des usages, à penser son travail comme rouage d'une conception interactive entre producteurs et usagers. Sa méthode consiste, en un premier temps, à «lire le destin de la mise en usage d'un nouveau produit ou service dans sa confrontation aux technologies "anciennes", aux pratiques existantes, à l'identité sociale et professionnelle de l'usager (place dans les enjeux, les imaginaires et les tactiques des divers usagers) et aux structures et aux évolutions d'ensemble de l'environnement social et professionnel de l'usager » (Froger \& Mallein 1997, 359). En un second temps, «si les concepteurs s'inspirent du savoir ainsi dégagé pour l'intégrer à leurs produits et services, alors ces derniers auront une excellente qualité d'usage, une valeur d'usage forte, qui favorisera un développement rapide de l'ingénierie concourante avec une technologie adaptée. S'ils ne le font pas, s'ils reproduisent par exemple la démarche d'imposition de technologies uniquement pensées par les ingénieurs concepteurs, alors ils auront toutes chances de mettre sur le marché des produits ou des services inadaptés à l'usage, sans signification d'usage pour une majorité, et courront un risque certain ».

Au moins Mallein est-t-il convaincu de « rendre service à la société » et de ne pas «trahir » son métier de chercheur. Beaucoup d'autres font ce passage au conseil moins professionnellement et moins ouvertement. Quoi qu'il en soit, cette « récupération » des études d'usage peut être lue de deux façons, les deux étant sans doute exactes. D'un côté, cette attention nouvelle aux usagers est une façon de les reconnaître dans leur capacité d'acteurs et non plus seulement de consommateurs passifs. Ce ne sont plus uniquement leurs «attentes » (ce qui était bien vague) qui sont prises en considération, mais surtout leurs «inventions » et leurs «réécritures» des produits ou des services. D'un autre côté, si l'imposition de types d'usages par les opérateurs s'estompe bien au bénéfice d'une réelle prise en compte d'un usager désormais tout puissant, le choix du développement des réseaux eux-mêmes n'est jamais posé comme enjeu de société. Ce développement est présenté comme «naturel» ou « inévitable ( voir le discours actuel sur Internet). Les avis des usagers sont l'objet de toutes les attentions tant qu'ils sont récupérables dans la perspective d'une augmentation des flux. Mais les raisons et les fins de cette augmentation ne sont jamais posées en tant que telles. La liberté d'usage et, mieux, la possibilité de pouvoir créer de nouvelles fonctionnalités ou horizons d'usages ne fait que renforcer l'impensé des choix techniques.

\section{Conclusion}

La sociologie des usages permet de dépasser l'image d'un usager buvard qui, selon sa porosité, absorberait avec plus ou moins de talent l'innovation technologique. En intervenant dans le quotidien même de cet usager et en observant ses manières de faire, cette sociologie l'a d'abord dégagé de ce rôle passif. En mesurant l'écart entre les usages effectifs des nouvelles machines à communiquer et les usages prescrits par leurs promoteurs, elle a ensuite permis de penser cet usager comme acteur. En repérant 
comment ses «déviances », ses «braconnages » et ses «détournements » étaient non seulement des réactions et des façons d'être, mais aussi des créations et des manières de faire, elle a posé la question du changement social à partir des usages des NTIC, la débarrassant au passage de tout technologisme. Cependant, cette sociologie — dont l'objet est finalement ce que Jacques Perriault (1989) a appelé la « logique de l'usage » - a toutefois ses limites. En effet, si elle permet de bien cerner les pratiques à une échelle microsociologique, elle ne parvient que rarement à prendre en compte le macrosociologique, c'est-à-dire à produire une connaissance sur les grands courants qui traversent notre société, et à montrer en quoi et comment les usages peuvent y renvoyer. Cette limite est repérable :

1) par l'oubli que l'innovation est déjà elle-même un objet social. La sociologie des usages a eu trop souvent tendance à considérer l'objet ou le dispositif dont l'usage était étudié comme un produit au départ exogène à la société. La sociologie de la traduction montre pourtant comment il y a, en amont de la diffusion, incorporation, hybridation entre solutions techniques et problèmes sociaux, et que l'innovation technologique dépend grandement des rapports de pouvoir qui structurent le social.

2) par son opposition trop systématique entre la technique et l'usager. Il en résulte un=face à face dans lequel la technique est souvent appréhendée dans sa logique propre (comme indépendante du social) et l'usager considéré dans son individualité et sa subjectivité. Ceci conduit d'une part à accentuer le premier travers (poser la technique en soi, comme extérieure à la société) et d'autre part à psychologiser à réduire le comportement des usagers à sa psychologie et à risquer de perdre de vue la détermination sociale des usages.

3) par la surestimation de l'autonomie de l'usager. En s'opposant au modèle déterministe, les études d'usage ont permis, c'est leur mérite, de faire apparaître la figure de l'usager comme acteur (Jauréguiberry 2003). Mais celui-ci est pourtant pris dans des structures qui limitent constamment ses capacités de résistance, de bricolage ou de détournement. Et si cette façon de faire est, comme le pense Michel de Certeau, une manière de ruser avec le pouvoir, encore faut-il nommer ce dernier et dire en quoi il pèse sur les modes d'usage, chose qu'un enfermement sur l'usager pris comme une monade ne permet pas.

En se focalisant trop sur les usages en soi, cette approche court donc le risque d'oublier l'amont et l'aval social des usages. Un bilan de l'ensemble des études de «l'école française des usages » fait heureusement apparaître que ce n'est jamais tout à fait le cas. Certaines études, se plaçant aussi en amont, mettent en effet l'accent sur les relations entre types d'usage et appartenances socioculturelles, entre usages organisationnellement situés et capacités stratégiques ou encore entre latitude d'usage et pouvoir économique et politique. D'autres, se situant aussi en aval, questionnent les usages dans leur capacité à influer sur le partage entre espace privé et espace professionnel, sur les formes d'organisation du travail, sur la gestion du temps et de l'espace, ou encore sur les rouages démocratiques dans un environnement d'immédiateté télécommunicationnelle.

\section{Références bibliographiques}

BABOULIN J.-C., GAUDIN J.-P. \& MALLEIN P. 1983 Le magnétoscope au quotidien : un demi-pouce de liberté, Paris, Aubier

BOULLIER D. 1984 “Usages du videotex et utopie techniciste”, Réseaux-6, 21-36 
BOULLIER D. 1992 Modes d'emploi : traduction et réinvention des techniques, in Gras et al. Sociologie des techniques de la vie quotidienne, 239-246

CARRE D. \& PANICO R. 1997 “De l'usage du marketing au marketing de l'usage : la finalité de la notion d'usage revisitée", in Actes du Premier colloque international Penser les usages, Arcachon, France Télécom, 241-249

CERTEAU M. de 1980 L'invention du quotidien-1 : Les arts de faire, Paris, UGE

CHAMBAT P. 1994 "Usages des TIC : évolution des problématiques", Technologies de l'information et société-6-3, 249-270

CLAISSE G. \& ROWE F. 1993 "Téléphone, communication et sociabilité : des pratiques différentiées", Sociétés contemporaines-14-15, 165-189

DIMMICK J., PATTERSON S. \& SIKAND J. 1996 "Personal Telephone Network : A Typology and Two Empirical Studies", Journal of Broadcasting and Electronic Media-40, 45-59

FROGER J. \& MALLEIN P. 1997 "Approche prospectiviste de l'ingénierie concourante", in Actes du colloque : Penser les usages, Arcachon, 358-365

GRAS A., MORICO C., POIROT-DELPECH S. \& SCARDIGLI V. 1994 Face à l'automate : le pilote, le contrôleur et l'ingénieur, Paris, Publications de la Sorbonne

HADDON L. éd. 1997 Communications on the move: the Experience of Mobile Telephony in the 1990s, Farsta, Telia AB

JAUREGUIBERRY F. 1989 Un Goût d'ubiquité. Usages sociaux du visiophone, rapport final de recherche, CNET-CNRS

JAUREGUIBERRY F. 2003 Les branchés du portable. Sociologie des usages, Paris, PUF

JOUËT J. 2000 “Retour critique sur la sociologie des usages”, Réseaux-100, 389-521

JUAN S. 1995 Les formes élémentaires de la vie quotidienne, Paris, PUF

LAULAN A.-M. 1985 La résistance aux systèmes d'information, Paris Retz

LIEBES T. \& KATZ E. 1990 The Export of Meaning : Cross Cultural Readings of Dallas, New York, Oxford University Press

LING R., JULSRUD T. \& KROGH E. 1997 "The Goretex Principle: the Hytte and Mobile Telephone in Norway", in Haddon L. éd. Communications on the move: the Experience of Mobile Telephony in the 1990s, 97-119

MALLEIN P. \& TOUSSAINT Y. 1994 "L'intégration sociale des TIC : une sociologie des usages", Technologie de l'information et société-6-4, 315-335.

MATTELART A. \& NEVEU E. 1996 “Cultural Studies Stories”, Réseaux-80, 11-58

MERCIER P.-A., PLASSARD F. \& SCARDIGLI V. 1984 La société digitale : les nouvelles technologies au futur quotidien, Paris, Seuil

MONJARET A. 1996 "Les communications téléphoniques privées sur les lieux de travail. Partage sexué des rôles", Traverse-72, 53-63 
PERRIAULT J. 1989 La logique de l'usage. Essai sur les machines à communiquer, Paris, Flammarion

PROMOVOST G. 1994 "Médias : éléments pour l'étude de la formation des usages sociaux", Technologie de l'information et société-6-4, 377-400

PROULX S. 2001 Usages des technologies d'information et de communication: reconsidérer le champ d'étude ?, in Actes du XXII ${ }^{\circ}$ Congrès national des sciences de l'information et de la communciation, 10-13 janvier 2001, 57-66

SCARDIGLIV. éd. 1993 L'Europe de la diversité: la dynamique des identités régionales, Paris, éditions du CNRS

SILVERSTONE R., MORLEY D., DALHBERG A \& al. 1989 "Families, Technologies and Consumption: the Household and Information and Communication Technologies", Program on Information and Communication Technologies Conference, Brunel University

TARRIUS A. 1994 “Entre sédentarité et nomadisme”, Réseaux-65, 59-70 